\title{
Triggered Convection, Gravity Waves, and the MJO: A Shallow-Water Model
}

\author{
DA YANG AND ANDREW P. INGERSOLL \\ Division of Geological and Planetary Sciences, California Institute of Technology, Pasadena, California
}

(Manuscript received 13 September 2012, in final form 18 February 2013)

\begin{abstract}
The Madden-Julian oscillation (MJO) is the dominant mode of intraseasonal variability in the tropics. Despite its primary importance, a generally accepted theory that accounts for fundamental features of the MJO, including its propagation speed, planetary horizontal scale, multiscale features, and quadrupole structures, remains elusive. In this study, the authors use a shallow-water model to simulate the MJO. In this model, convection is parameterized as a short-duration localized mass source and is triggered when the layer thickness falls below a critical value. Radiation is parameterized as a steady uniform mass sink. The following MJO-like signals are observed in the simulations: 1) slow eastward-propagating large-scale disturbances, which show up as low-frequency, low-wavenumber features with eastward propagation in the spectral domain, 2) multiscale structures in the time-longitude (Hovmöller) domain, and 3) quadrupole vortex structures in the longitudelatitude (map view) domain. The authors propose that the simulated MJO signal is an interference pattern of westward and eastward inertia-gravity (WIG and EIG) waves. Its propagation speed is half of the speed difference between the WIG and EIG waves. The horizontal scale of its large-scale envelope is determined by the bandwidth of the excited waves, and the bandwidth is controlled by the number density of convection events. In this model, convection events trigger other convection events, thereby aggregating into large-scale structures, but there is no feedback of the large-scale structures onto the convection events. The results suggest that the MJO is not so much a low-frequency wave, in which convection acts as a quasi-equilibrium adjustment, but is more a pattern of high-frequency waves that interact directly with the convection.
\end{abstract}

\section{Introduction}

The Madden-Julian oscillation (MJO) is the dominant intraseasonal variability in the tropical atmosphere. It is a planetary-scale, slow eastward-propagating (about $5 \mathrm{~m} \mathrm{~s}^{-1}$ ) perturbation of both dynamical and thermodynamical fields. During an MJO event, a positive convection and rainfall anomaly develops in the western Indian Ocean and propagates to the western Pacific Ocean. Once the perturbation reaches the date line, the perturbation is largely confined to dynamical fields. The associated planetary-scale wind structure is baroclinic and is characterized by low-level convergence and upper-level divergence (Madden and Julian 1972, 1994; Hendon and Salby 1994). More details can be found in comprehensive reviews such as Madden and Julian (2005) and Zhang (2005). In addition, within the MJO envelope, there are both westward- and eastward-moving finescale

Corresponding author address: Da Yang, Division of Geological and Planetary Sciences, MC 150-21, California Institute of Technology, Pasadena, CA 91125.

E-mail: dyang@caltech.edu structures (Nakazawa 1988; Hendon and Liebmann 1994).

The significance of the MJO for phenomena such as monsoon onset, ENSO, and rainfall patterns in the tropics has been well established (Zhang 2005). However, a successful MJO theory is elusive. For a historical and detailed review of theories, please refer to Zhang (2005) and Wang (2005). Here we will review three schools of theories. One school considers the MJO as a large-scale unstable mode in the tropics, and it is often referred to as the moisture mode. The moisture mode arises from positive feedbacks between precipitation and the source of moist static energy (e.g., Neelin and Yu 1994; Sobel et al. 2001; Fuchs and Raymond 2002, 2005; Bretherton et al. 2005; Fuchs and Raymond 2007; Maloney 2009; Raymond and Fuchs 2009; Andersen and Kuang 2012). Another school still considers the MJO as a large-scale mode, but the major instability to maintain the planetary-scale envelope happens in the synoptic scales. Both observational and modeling studies suggest that high-frequency small-scale waves are important to the MJO (e.g., Hendon and Liebmann 1994; Tung and Yanai 2002; Moncrieff 2004; Biello and Majda 2005; 
Khouider et al. 2012). Thus, Majda and Stechmann $(2009,2011)$ emphasize the importance of small-scale waves within the MJO envelope, but they parameterize the effect of the waves. In their model, it is only the amplitude of the wave activity envelope that is needed, not any of the details of the particular synoptic-scale waves that make up the envelope. The third school considers the $\mathrm{MJO}$ as a wave packet of a certain type of equatorial waves, and the MJO propagates with the group velocity of the equatorial waves. Yang and Ingersoll (2011) hypothesize that the MJO is a mixed Rossby-gravity (MRG) wave packet that propagates with the MRG group velocity, and they test this hypothesis, both with an idealized general circulation model (GCM) and with the outgoing longwave radiation (OLR) dataset. They force with a westward-moving heat source that lasts for 10 or 20 days. At the same time, Solodoch et al. (2011) suggest that the MJO could be a forced response to the MRG wave group in the quasiequilibrium (QE) convection and wind-induced surface heat exchange context. Neither theory considers inertiagravity (IG) waves, which have frequencies greater than $0.5 \mathrm{cpd}$. In the QE context, these high-frequency, smallscale waves will be damped the fastest, and it is possible that the QE approach does not apply there. Therefore, the role of high-frequency, small-scale waves has not really been investigated in any of the theories, including the wave packet theories of the MJO. Here we present a simple MJO model with triggered (non-QE) convection that emphasizes the multiscale structures of the MJO.

General circulation models (GCMs) simulate tropical intraseasonal variability with varying degrees of fidelity. Intercomparison studies (Lin et al. 2006) show that most GCMs underestimate the MJO variance. The weak MJO signals in GCMs are believed to be caused by inadequate convection schemes in the GCMs. Holloway et al. (2012) compare limited-area simulations of the tropical atmosphere over a very large domain at different horizontal resolutions with both parameterized and explicit convection versions for a 10-day MJO case study in April 2009. They claim that the parameterized models consume convective available potential energy (CAPE) and reach radiative-convective equilibrium too quickly and that they lack the ability to transition from suppressed to active conditions and vice versa. As a result, propagating MJO signals are absent in the parameterized runs.

There are two common ways of treating convection: triggered and QE convection. There is not a clear distinction between these two categories. Over a long period, in the tropics, the generation of CAPE by largescale processes nearly balances its consumption by convection. The convection may be considered to be in a state of statistical equilibrium with the large-scale circulation. The QE idea was first applied by Arakawa and Schubert (1974). This idea has been validated using observational datasets by Xu and Emanuel (1989) and Holloway and Neelin (2007). QE is a good assumption for studying large-scale circulations that vary slowly with time compared with convective time scales. Such circulations include tropical cyclones, the Hadley cell, and monsoon circulations (e.g., Emanuel et al. 1994; Emanuel 2007), but so far the MJO has not been successfully simulated under the QE context. If we are interested in high-frequency, small-scale waves, however, QE does not work well. First, over a short period, CAPE builds up. When some threshold is reached, convection is triggered and CAPE is released. Second, in a QE scheme, convection will damp small-scale waves faster and leave the large-scale waves.

Recent studies (Benedict and Randall 2009) with a superparameterized (SP) Community Atmosphere Model (CAM) show improved MJO simulations. The SP CAM replaces conventional boundary-layer, moist-convection parameterizations with a cloud-resolving model embedded in each CAM grid cell (Khairoutdinov and Randall 2001). The SP CAM deals with subgrid-scale variability more accurately. Our interpretation of the SP CAM results is that the cloud-resolving model has a continuous spectrum from the triggered convection to statistical equilibrium convection, such that it treats the highfrequency, small-scale waves, which are crucial to the MJO, equally well as the low-frequency, large-scale variability.

Motivated by the success of SP CAM simulations, we develop a theory emphasizing the role of high-frequency, small-scale waves. In this paper, we use a shallow-water model with triggered convection and radiation represented as Newtonian relaxation. Slow eastward-propagating (MJO-like) signals are observed in our simulations. Instead of thinking of the MJO as a large-scale stable or unstable mode, we propose that the MJO-like signals are interference patterns of westward and eastward inertiagravity (WIG and EIG) waves that interact directly with the convection. Eastward propagation is due to the zonal asymmetry of the EIG and WIG waves. In section 2, we will introduce the shallow-water model used in this study. In sections 3 and 4 we show our simulation results and attempts to understand the simulated signal. In section 5, we will discuss our results and present our conclusions and future work.

\section{Model description}

We use a $2 \mathrm{D}$ shallow-water model in this study. We simulate the upper troposphere by assuming the first baroclinic mode, since the large-scale circulation associated with the MJO shows the first baroclinic structure. 
Thus, divergence in the model refers to upper-level divergence and low-level convergence. Similarly, large layer thickness in the model corresponds to high pressure aloft and low pressure near the surface. In this section, we will introduce the shallow-water model used in this study.

This shallow-water model describes the evolution of constant density, incompressible fluid over the surface of the sphere. The model equations are

$$
\begin{aligned}
& \partial_{t} u=f v-\frac{u}{a \cos \theta} \partial_{\lambda} u+\frac{v}{a} \partial_{\theta} u+\frac{u v \tan \theta}{a}-\frac{1}{a \cos \theta} \partial_{\lambda} \phi, \\
& \partial_{t} v=-f u-\frac{u}{a \cos \theta} \partial_{\lambda} v+\frac{v}{a} \partial_{\theta} v+\frac{u^{2} \tan \theta}{a}-\frac{1}{a} \partial_{\theta} \phi, \\
& \partial_{t} \phi=-\nabla \cdot(\mathbf{V} \phi)+q-r .
\end{aligned}
$$

Equations (1) and (2) are momentum equations, where $u$ and $v$ are zonal and meridional velocities; $\phi$ is the geopotential, which is gravity $g$ times the equivalent layer thickness (equivalent depth); $a$ is the earth radius; $f$ is the Coriolis parameter (also known as planetary vorticity); and $\lambda$ and $\theta$ represent longitude and latitude in radians. Equation (3) is the continuity equation, where $q$ represents convective heating, which is a mass source, and $r$ represents radiative cooling, which is a mass sink.

In this model, convection events are triggered by a low value of the layer thickness, that is, if the layer thickness is lower than a threshold $\phi_{c}$, convection will start to add mass into this shallow-water system. This trigger mechanism can be related to mass accumulation in the lower troposphere. In this model, we assume the first baroclinic mode and simulate the upper troposphere. A low value of $\phi$ is equivalent to an accumulation of mass and moisture in the lower troposphere. This is an environment that favors convection. The convective heating is given by

$$
q= \begin{cases}\frac{q_{0}}{\tau_{c} A_{0}}\left[1-\left(\frac{\Delta t-\tau_{c} / 2}{\tau_{c} / 2}\right)^{2}\right]\left(1-\frac{L^{2}}{R^{2}}\right) & \text { when } \phi<\phi_{c}, 0<\Delta t<\tau_{c}, \quad \text { and } L^{2} \leq R^{2} \\ 0 & \text { otherwise }\end{cases}
$$

where $q_{0}$ is a free parameter of the heating amplitude, $\tau_{c}$ is the convective time scale, and $\Delta t$ is measured relative to the time when convection is triggered. Each convection event operates in a certain area $A_{o}=\pi R^{2}$, where $R$ is the radius of each convection event. The equation $L=\left(\Delta x^{2}+\Delta y^{2}\right)^{1 / 2}$ measures the distance from the convective center, where $\Delta x$ and $\Delta y$ are measured relative to the location where the convection is triggered.

The radiative cooling is constant in both time and space. The ratio $3 r / q_{o}$ determines $\dot{\Sigma}$, the rate of initiation of convection events per unit area per unit time. In a statistically steady state, the total mass of this system will not change with time. Convection, the mass source, will be balanced by the mass sink, radiation. The equilibrium geopotential is approximately $\phi_{c}$.

The shallow-water equations have characteristic length and time scales through the planetary radius and the rotation. In addition to the planetary radius and rotation, there are four parameters: the equilibrium geopotential $\phi_{c}, A_{o}, \tau_{c}$, and $\dot{\Sigma}$, which is controlled by $r$ through the relation $\dot{\Sigma} \sim 3 r / q_{o}$. Both $q_{o}$ and $r$ are small, so the fluid dynamics in our model are linear. Therefore, the forcing amplitudes are not free parameters and only the ratio between the two terms matters. The forcing is through $q$ and $r$, and they cause $\phi$ to fluctuate around $\phi_{c}$, which appears in Eq. (4). So for constant $r / q_{o}$, the three

variables $u, v$, and $\phi-\phi_{c}$ all scale as $r$, which can be arbitrarily small. We can scale $r$ and the other variables by any small number, then run the model, undo the scaling, and the results are statistically the same. Therefore, the absolute value of our model output is not important. Only the relative magnitude matters.

Scaling is one property of linear systems, but our model is not linear. It does not satisfy superposition. The model has a given climatology, which is independent of the initial conditions. If we superpose two sets of initial conditions and then run the model, we get a different answer than we would get by superposing the two solutions after running the model. The nonlinearity does not come from the advection term, since the scaled amplitude can be arbitrarily small. Instead, it comes from the forcing terms $r$ and $q$ and the threshold condition in Eq. (3).

The Kelvin wave speed $c$ is equal to $\sqrt{\phi}_{c}$. In this study, we fix $c \sim 16 \mathrm{~m} \mathrm{~s}^{-1}$. The mean depth $h_{e}$ (equivalent depth) of this SW system is given by $\phi_{c} / g$. In our control simulation, $R$ is $3^{\circ}$ of latitude, which is approximately the size of the grid in T42 simulations. Parameter values of our control simulation are documented in Table 1. We vary the horizontal resolution from T42 to T170 and find our main results are not sensitive to resolution. The results presented in this paper are mainly from $\mathrm{T} 42$ 
TABLE 1. Parameter values in the control simulation.

\begin{tabular}{cccc}
\hline \hline$\phi_{c}\left(\mathrm{~m} \mathrm{~s}^{-1}\right)^{2}$ & $R_{o}\left({ }^{\circ}\right)$ & $\tau_{c}$ (day) & $\dot{\Sigma}_{o}\left(\mathrm{~m}^{-2} \mathrm{~s}^{-1}\right)$ \\
\hline 250 & 3 & 0.25 & $1.12 \times 10^{-17}$
\end{tabular}

simulations. We solve these equations in spherical coordinates by using the spectral dynamical core of the Geophysical Fluid Dynamics Laboratory (GFDL) Flexible Modeling System. Each simulation is initialized with random noise. Although we do not have a moisture variable explicitly in our model, our model does illustrate the importance of moisture to the MJO.

\section{Simulation results}

Figure 1 shows Hovmöller diagrams of our shallowwater simulation from day 500 to day 600 . Figure 1 shows the symmetric components of the geopotential, zonal wind, and convective heating and the antisymmetric components of meridional wind. The symmetry is with respect to the equator. Such meridional symmetry excludes the even meridional-wind modes and leaves the odd modes. Figure 1a shows the geopotential. There are two major large-scale events labeled A and B. These are the MJO-like signals. They move eastward at approximately $3.0 \mathrm{~m} \mathrm{~s}^{-1}$. Small-scale waves are present, and they include Kelvin waves, IG waves, and Rossby waves. Since the IG waves are small-scale waves, the absolute values of their speed are close to the Kelvin wave speed, which is approximately $16 \mathrm{~m} \mathrm{~s}^{-1}$. Figure $1 \mathrm{~b}$ shows the zonal wind. The white represents westward zonal wind, and the black represents eastward zonal wind. The edge between the white and black indicates the divergence of zonal wind. Two regions of large-scale divergence are observed, and they are collocated with events A and B in Fig. 1a. Figure
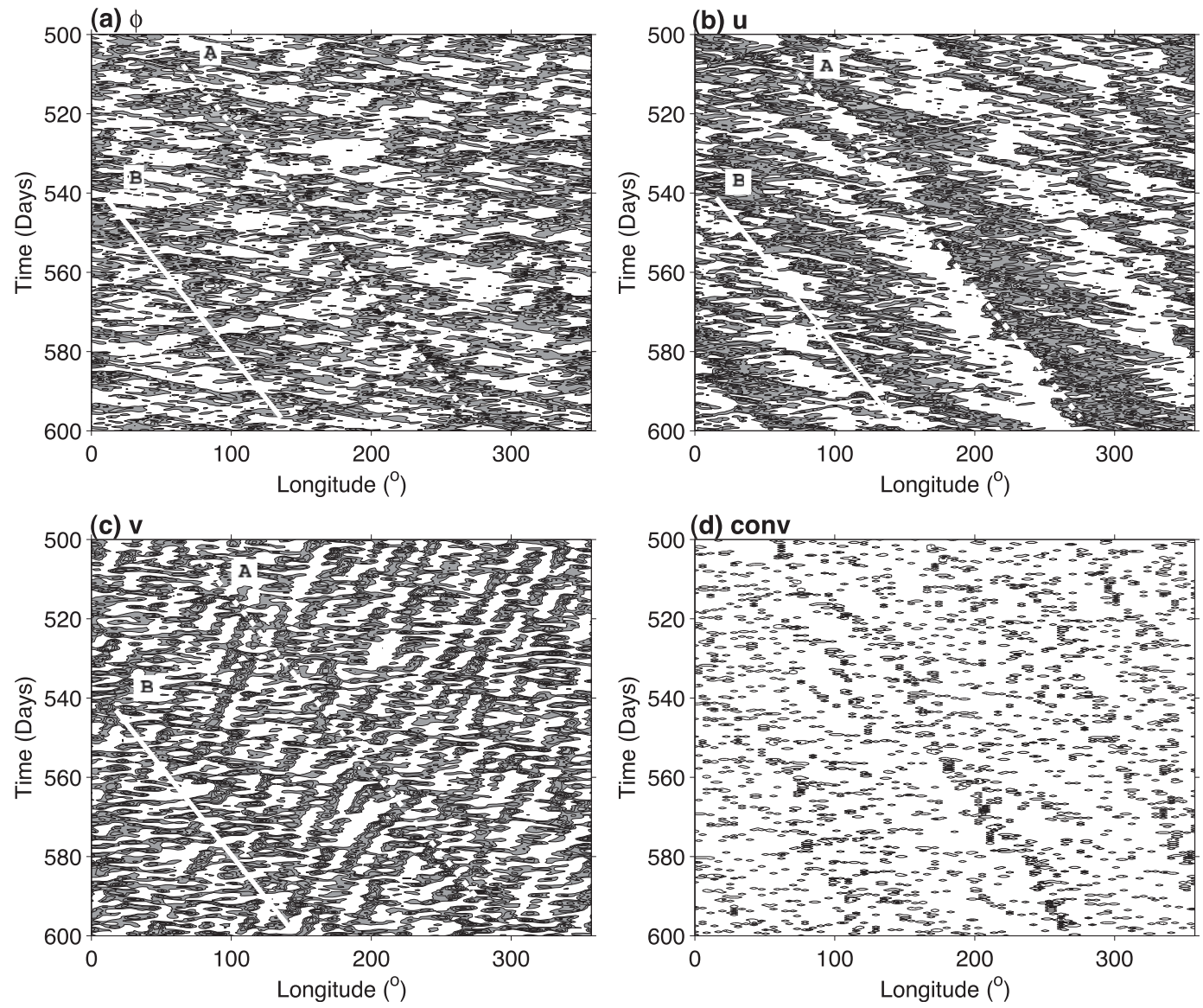

FIG. 1. Hovmöller diagrams of the (a) geopotential, (b) zonal wind, (c) meridional wind, and (d) convective heating of our shallow-water simulation from $-15^{\circ}$ to $15^{\circ}$ latitude. The geopotential, zonal wind, and convective heating are symmetric components about the equator, and the meridional wind is the antisymmetric component. The white (black) represents low (high), and the contour interval is linear. The forcing amplitude is arbitrarily small. Therefore, the absolute value of our model output is not important. The dashed and solid lines represent MJO events A and B, respectively, and the propagation speeds are both about $3.0 \mathrm{~m} \mathrm{~s}^{-1}$. 
(a) $u$

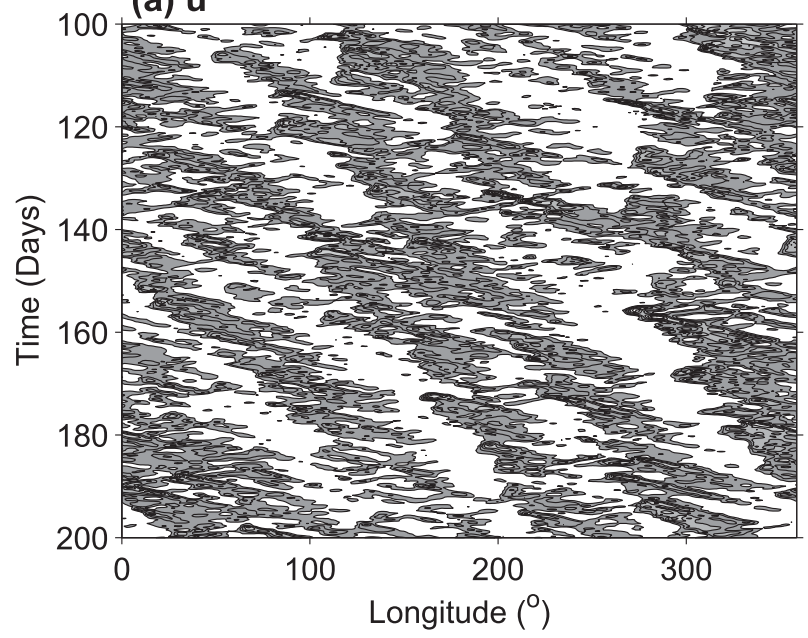

(b) $u$

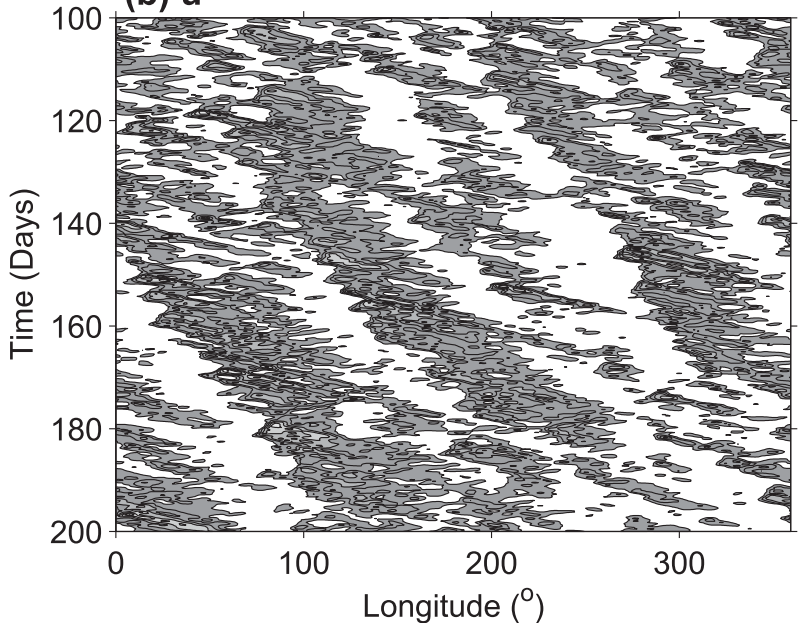

FIG. 2. Hovmöller diagrams of zonal wind from (a) T85 and (b) T170 simulations. Colors and contour intervals are as in Fig. 1 b.

1c shows $v$. Small-scale westward and eastward waves are observed, but large-scale envelopes are not clear in the $v$ field. Figure 1d shows convective heating. Convection is a small-scale, short-duration process, but two organized, long-lasting events are observed, and they are collocated with events A and B in Fig. 1a and with large-scale divergence in Fig. 1b. Similar to the observation, large-scale divergence is collocated with convective centers, and dynamical fields are coupled to convection.

Figure 2 shows the Hovmöller diagrams of the zonal wind from T85 and T170 simulations with the same parameters as the T42 simulations of Fig. 1b. The size of the convection in kilometers is the same. They both show propagation speeds and horizontal structures that are similar to the T42 simulations. This comparison suggests that the T42 simulations have already converged. When $R$ is $3.0^{\circ}$, convection is not well resolved in T42 simulations, and this induces discontinuities between grid points.
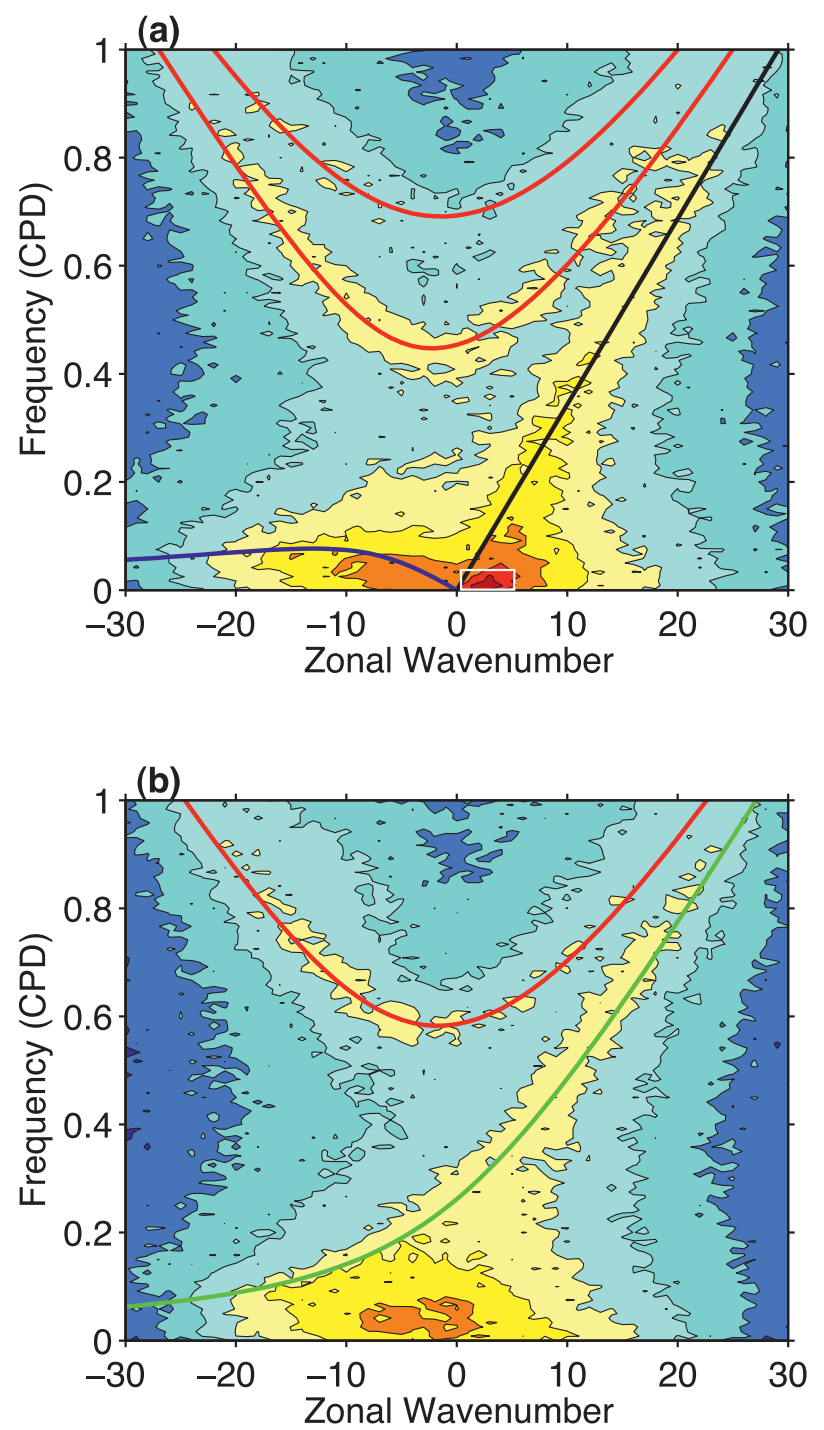

FIG. 3. Zonal wavenumber-frequency power spectra of zonal wind from $-15^{\circ}$ to $15^{\circ}$ latitude for (a) symmetric and (b) antisymmetric components. Red represents high-power density, and blue represents low-power density. Red, blue, black, and green lines denote dispersion curves of IG, Rossby, Kelvin, and MRG waves, respectively, for different meridional modes. The white box in (a) ranges from 1 to 5 in wavenumber and from $1 / 60$ to $1 / 200 \mathrm{cpd}$ in frequency. The logarithm to the base 10 is taken for plotting, and the contour interval is 0.5 .

However, such discontinuity can be smoothed out by hyperviscosity. As a result, T42 simulations produce similar results to T85 and T170 simulations, where convection is well resolved. In the rest of the paper, the simulation results are from the T42 simulations, unless otherwise noted.

To understand the multiscale structures in our simulation, we carried out space-time spectral analysis as pioneered by Wheeler and Kiladis (1999, hereafter WK99). Figures $3 a$ and $3 b$ show the symmetric and 


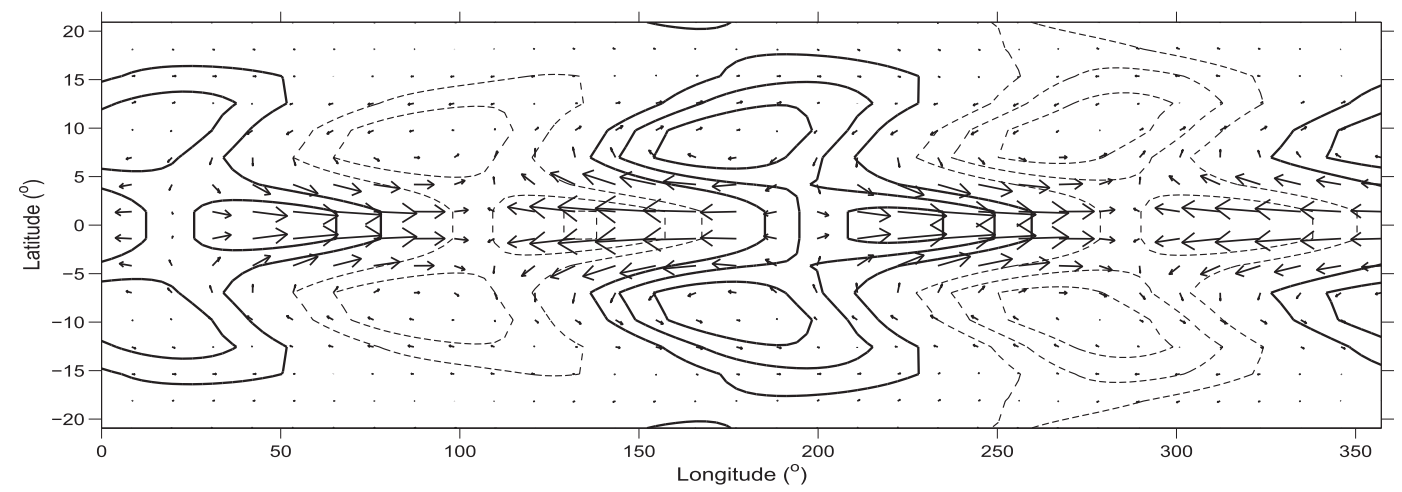

FIG. 4. Longitude-latitude map of composites of the MJO-like signals. The arrows denote the wind field $\mathbf{V}=(u, v)$, and the contours denote the geopotential. The thick solid (thin dashed) contours represent positive (negative) anomalies with linear contour intervals.

antisymmetric components (about the equator) of the zonal wind, respectively. Superimposed curves represent dispersion relations of equatorial waves for an assumed $16 \mathrm{~m} \mathrm{~s}^{-1}$ Kelvin wave speed. The dispersion curves of the equatorial waves were first derived by Matsuno (1966). Different equatorial waves are characterized by different dispersion relations and meridional mode numbers $n$. The spectral power under the superimposed curves is associated with the corresponding equatorial waves. In Fig. 3a, we can see spectral peaks associated with the $n=1$ Rossby wave, the Kelvin wave, and the $n=1$ and $n=3$ IG waves. Consistent with the slow eastward-moving signals in Fig. 1, Fig. 3a has an MJO-like signal within the white box. This signal has planetary scale and low frequency. In the rest of this paper, we will try to understand this interesting phenomenon. In Fig. 3b, we can see spectral peaks associated with the $n=2$ Rossby wave, the MRG wave, and the $n=2 \mathrm{IG}$ wave. A striking feature of Fig. 3 is the intense power associated with high-frequency IG waves. High IG wave activity is associated with the convective parameterization in our model. We will argue that high IG wave activity is the key to the MJO-like signal. Nevertheless, most of the power is concentrated in low-wavenumber and low-frequency regions. This is, in general, a red spectrum. Although different in details, Fig. 3 captures some fundamental features of the observed spectra by WK99.

Figure 4 shows the horizontal structure of the MJOlike signal in our simulation. To get Fig. 4, we take the MJO-filtered signal of the zonal wind in the wavenumber-frequency domain. The filtering excludes all wavenumbers and frequencies except those in the MJO box (Fig. 3a). Following Wheeler and Hendon (2004), we carried out empirical orthogonal function (EOF) analysis of the MJO-filtered signal. We found the first two EOFs can contribute about $85 \%$ of the total variance of the MJO-filtered signal. The EOFs together with the corresponding principal components (PCs) show the propagation behavior of the MJO-like signal. Combining all the phases of the MJO-like signal, we get the horizontal structure shown in Fig. 4. The contours represent the geopotential, and the vectors represent the wind. Away from the equator we can see cyclonic and anticyclonic vortices. At the equator, the wind is more zonal, and the contours are more parallel to the equator. In this figure, a wavenumber-2 pattern stands out, that is, the zonal wind alternates from eastward to westward twice in the domain. Consider one cycle of the pattern, that between $100^{\circ}$ and $280^{\circ}$ longitude. The maximum divergence of the zonal wind occurs at $180^{\circ}$. To the west of the maximum divergence, at about $160^{\circ}$, there are anticyclones to the north and the south, collocated with high geopotential anomalies. To the east of the divergence of the zonal wind, there are cyclones centered at about $10^{\circ}$ latitude, together with low geopotential anomalies. This structure is referred to as the quadrupole vortex structure (Majda and Stechmann 2009). The quadrupole vortices in our simulations are confined more closely to the equator than in the MJO as reported by Kiladis et al. (2005), where the off-equatorial vortices center at about $20^{\circ}$ latitude.

\section{Proposed mechanism}

MJO-like signals have been simulated in our model. The simulated signal captures major features of the MJO, including propagation speed, horizontal scale, multiscale structures, and quadrupole vortex structures. The next step is to understand the slow eastward propagation and the low wavenumber of the disturbance. Figure 5 shows high-resolution Hovmöller diagrams. In this high-resolution view, we are able to diagnose how convection is triggered and how waves are excited. In Fig. 5a, there is a standing oscillation at a longitude of 

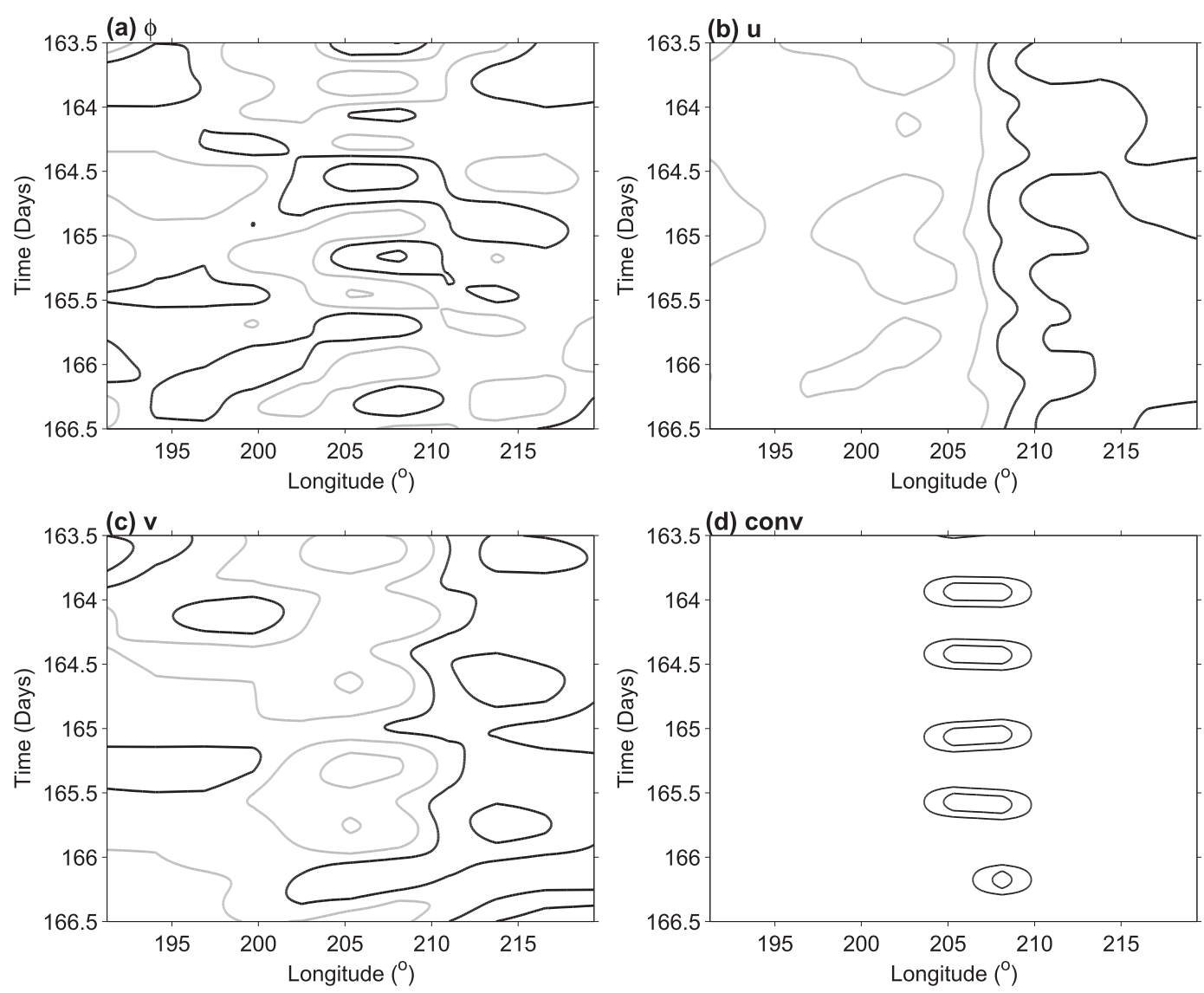

FIG. 5. High-resolution Hovmöller diagrams of the (a) geopotential, (b) zonal wind, (c) meridional wind, and (d) convective heating of our shallow-water simulation from $-6^{\circ}$ to $6^{\circ}$ latitude. The geopotential, zonal wind, and convective heating are symmetric components about the equator, and the meridional wind is the antisymmetric component. The black (gray) line denotes positive (negative) anomalies with linear contour intervals.

about $206^{\circ}$. On day 163.75 , there is a local minimum of $\phi$ at $206^{\circ}$. This triggers a convection event. A quarter cycle later, the convection reaches its maximum value, and a quarter cycle after that, $\phi$ reaches a local maximum. Half a cycle later, $\phi$ is a local minimum and another convection event is triggered. Such cycles repeat five times in Fig. 5 with a period of 0.5 day, which is twice $\tau_{c}$. The amplitude of convective heating is small, so there is no nonlinearity due to advection. Standing waves are excited, and their frequency is approximately $1 / 2 \tau_{c}$. We call this the quasi frequency. Convection excites IG waves at this frequency, but it excites waves at other frequencies, including zero, as well. The series of convection events shown in Fig. 5 is like the top half of a truncated sine wave. Many frequencies are present in this function. Thus, energy is introduced into this shallow-water system. The standing oscillation can be viewed as a superposition of eastward and westward waves with similar speeds. In the power spectra, WIG and EIG waves are the only waves that propagate toward each other with the same meridional structures and similar propagation speed. Therefore, the simulated MJO signal is an interfering pattern between WIG and EIG waves, which are excited by convection. The propagation speed of the MJO pattern is associated with the phase speed difference between WIG and EIG waves.

Figure 6 shows how our hypothesis could explain the MJO propagation speed quantitatively. If the hypothesis is correct, the MJO propagation speed is one-half of the phase speed difference between the WIG and EIG waves. In Eq. (5), the EIG phase speed is $c_{1}$, the WIG phase speed is $c_{2}$, and the cosine factor on the right-hand side is the standing oscillation. The sine factor on the right-hand side is the drift of the pattern at the MJO propagation speed:

$$
\begin{aligned}
& \sin \left[k\left(x-c_{1} t\right)\right]+\sin \left[k\left(x+c_{2} t\right)\right] \\
& \quad=2 \sin \left[k\left(x-\frac{c_{1}-c_{2}}{2} t\right)\right] \cos \left[k \frac{\left(c_{1}+c_{2}\right) t}{2}\right] .
\end{aligned}
$$




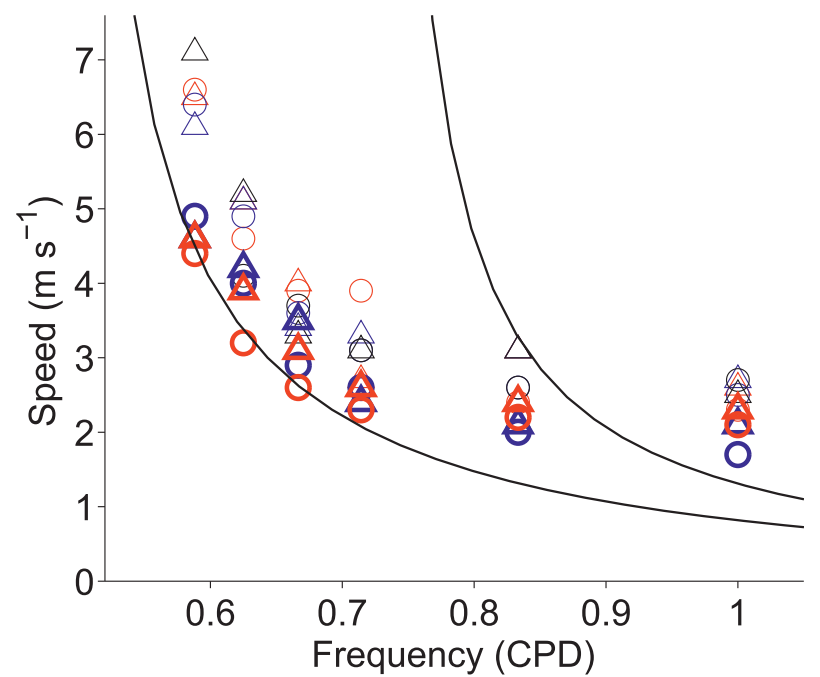

FIG. 6. Propagation speed $\left(\mathrm{m} \mathrm{s}^{-1}\right)$ vs quasi frequency (cpd). The lower and upper solid lines are derived from the dispersion relations of the $n=1$ and $n=3$ IG waves, respectively. The markers represent the simulation results for different parameters. The thicker markers are for the T170 simulations, and the thin ones are for T85 simulations. The markers are red when $\dot{\Sigma}=\dot{\Sigma}_{o}$, blue when $\dot{\Sigma}=0.5 \dot{\Sigma}_{o}$, and black when $\dot{\Sigma}=0.25 \dot{\Sigma}_{o}$. The triangles are for simulations when $R=R_{o}$, and the circles are for simulations when $R=$ $0.5 R_{o}$, where $R_{o}=3.0^{\circ}$. For example, a thick red circle is from the T170 simulation with $\dot{\Sigma}=\dot{\Sigma}_{o}$ and $R=0.5 R_{o}$. We convert $\tau_{c}$ to frequency by using frequency equals $1 / 2 \tau_{c}$. The values of $R_{o}$ and $\dot{\Sigma}_{o}$ are given in Table 1.

The solid line denotes the theoretically derived MJO propagation speed from the dispersion relation of the two lowest symmetric IG waves, with $n=1$ and $n=3$. The markers represent simulation results with different parameters. The abscissa is the quasi frequency of IG waves. We have systematically varied the parameters in our model, including $R, \tau_{c}, \dot{\Sigma}$, and the model resolution (Table 2). There is a finite spread in speed when fixing $\tau_{c}$ and changing other parameters. However, simulation results show consistent behavior when varying $\tau_{c}$. The lower solid curve, corresponding to $n=1$, fits our simulation results relatively well, especially for the T170 simulations. At the high-frequency end of this plot, the simulated speeds seem to level off at $2 \mathrm{~m} \mathrm{~s}^{-1}$, which corresponds to the quasi-standing wave speed at a frequency of about $0.7 \mathrm{cpd}$. As shown in Fig. 3a, this is the minimum frequency of the $n=3$ mode. The $n=1$ mode fits the numerical model below this frequency because it is the only mode that can exist. At frequencies above $0.7 \mathrm{cpd}$, convection excites both the $n=1$ mode and the $n=3$ mode. At still higher frequencies, convection will excite even higher meridional modes, and no single mode will match the numerical results.

Another reason the propagation speeds level off at high frequencies (Fig. 6) is that convection might
TABLE 2. Parameter ranges in Fig. 6.

\begin{tabular}{cccr}
\hline \hline$R\left(^{\circ}\right)$ & $\tau_{c}$ (day) & $\dot{\Sigma}\left(10^{-18} \mathrm{~m}^{-2} \mathrm{~s}^{-1}\right)$ & Resolution \\
\hline $1.5-3.0$ & $0.5-0.85$ & $2.8-11.2$ & T85-T170 \\
\hline
\end{tabular}

be exciting quasi-standing waves at frequencies below $1 / 2 \tau_{c}$. This could happen because the convection is not a single-frequency signal and has a dc component. Because of the positive-only convective heating, there is a net heating at one place that persists over several cycles of the wave-a time period longer than $2 \tau_{c}$. As a result, waves with frequency lower than $1 / 2 \tau_{c}$ will be excited and possibly amplified. This is why we call $1 / 2 \tau_{c}$ the quasi frequency rather than the forcing frequency. The main conclusion from Fig. 6 is that the propagation speed of the MJO-like disturbance is that of the quasistanding IG wave. The evidence for this statement is strongest at frequencies below $0.7 \mathrm{cpd}$, where only the $n=1$ wave can be excited. At those frequencies, the quantitative agreement is quite good. At higher frequencies there is a mixture of waves, and it is harder to see the effects of any one wave.

As an interference pattern, the horizontal scale of the MJO will be inversely related to the bandwidth of the excited waves-the number of zonal wavenumbers that are excited. Our simulations show that the bandwidth of waves is associated with how many convection events are happening at the same time, that is, $\dot{\Sigma}$. If $\dot{\Sigma}$ is large, many waves with various frequencies are excited and the bandwidth is large. However, if there are only a few convection events during the simulation, only a few waves are excited and the bandwidth is small. Because of the dc component of the heating, the low frequencies are related to the persistence of the gravity wave rather than to its period. With many convection events happening at once, the phase and amplitude of the gravity waves are constantly changing. The heating does not persist at any one place, and the low frequencies do not develop. Since the convection rate $\dot{\Sigma}$ is of order $3 r / q_{o}$, increasing $r$ will increase $\dot{\Sigma}$ if all the other parameters are fixed. As a result, the MJO wavenumber increases. Figure 7 shows the results of systematic experiments where the MJO wavenumber increases as $r$ increases. The amplitude of the convective forcing is small so that the waves are linear waves, and decreasing the convective strength does the same job as increasing $r$.

One must ask, how does convection, which is a small-scale, short-duration event, generate a large-scale, low-frequency response in our shallow-water system? The answer is that convection excites a range of frequencies, including zero frequency since convection has a dc component, given by the threshold behavior of 


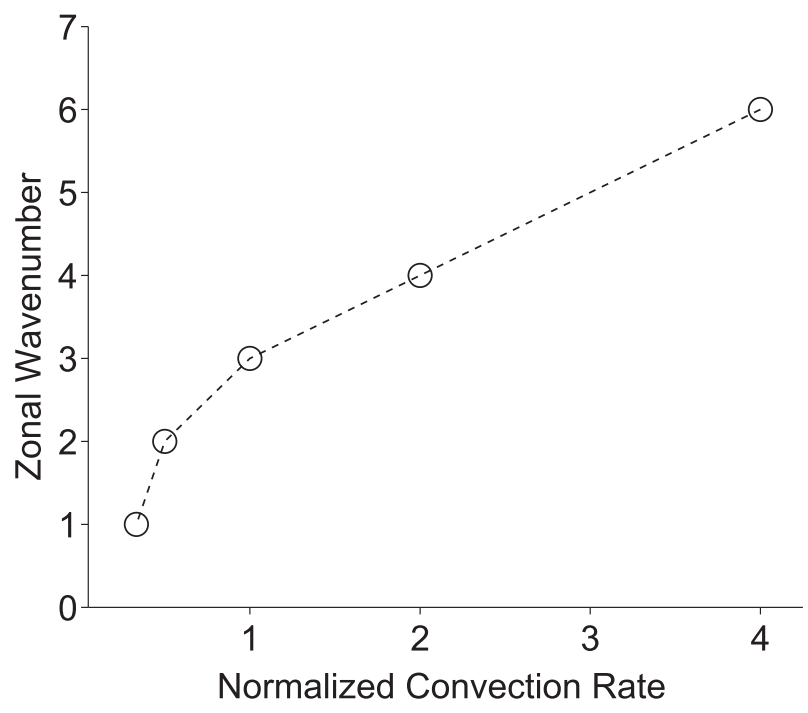

FIG. 7. Zonal wavenumber vs normalized convection rate $\dot{\Sigma} / \dot{\Sigma}_{o}$, where $\dot{\Sigma}_{o}$ is given in Table 1 .

convection. It may appear strange that a model forced at short time scales is giving rise to oscillations at low frequencies, especially since the advection terms are negligible. But the model is not externally forced: it is self-excited. Convection excites a quasi standing IG wave, which triggers more convection events in the vicinity of recent convection events. In the statistically steady state, one has a large-scale, slowly varying aggregation of convection events. In our model, the large-scale pattern does not directly affect the convection events, which have an intrinsic tendency to self-aggregate through exciting quasistanding IG waves. However, the convection has a dc component-it is positive only-so the envelope of convection events has a large-scale effect on the dynamics. Figure 1 provides an illustration. Convection is organized in events $\mathrm{A}$ and $\mathrm{B}$, and $\dot{\Sigma}$ is larger within the MJO envelopes. The organized convection along events $\mathrm{A}$ and $\mathrm{B}$ in Fig. 1d constantly puts mass into the shallow-water system while propagating eastward. This propagating mass source creates a large-scale eastward-propagating divergence (Fig. 1b). The envelopes of both the convection and the divergence have dc components. The dc components occupy a large horizontal area and persist for a long time. Therefore, the interference pattern of IG waves shows its unique dispersion character-low frequency and low wavenumber. The MJO structure in Fig. 4 is constructed from the low-frequency, lowwavenumber components in the Fourier domain. Therefore, it should show the large-scale response to the dc component of the convection envelope. That is why the MJO composites exhibit the quadrupole vortex structure instead of the IG wave structures.
We have done simulations with different sizes of convection by varying $R$ from $0.7^{\circ}$ to $3.0^{\circ}$ with the T170 resolution. Both the propagation speed and the horizontal scale of the MJO do not show significant dependence on $R$. We have also varied the hyperviscosity by an order of magnitude from the reference value, $1.0 \times 10^{-4} \mathrm{~m}^{8} \mathrm{~s}^{-1}$, and the simulation results are still robust. The redness of the power spectrum is not a result of the hyperviscosity. Instead, it results from the persistence of the quasi standing wave and the convection that it triggers. The large scale is related to the large size of the envelope, which is the interference pattern of the IG waves.

\section{Discussion and conclusions}

In this paper, we have presented a shallow-water model with triggered convection and simple radiation treatments. MJO-like signals are observed in our simulations. We propose that the MJO-like signal is an interference pattern of the WIG and EIG waves, whose frequency is set by the duration of individual convection events. Our simulation results suggest that the MJO is not a large-scale, low-frequency wave in which convection acts as a QE adjustment. Small-scale, high-frequency waves might be crucial. Therefore, in order to simulate the MJO, the behavior of moist convection in a short time period should be represented properly.

Moisture is implicit in our model. Triggered convection only occurs in a moist atmosphere, where conditional instability can exist. In a shallow-water model, the geopotential $\phi$ is a measure of the static stability $N^{2}$ of the atmosphere. Low $\phi$ indicates reduced $N^{2}$. Only if $N^{2}$ has been reduced to a critical value, in other words, CAPE has been accumulated by a certain amount, will convection be triggered. This mimics the processes of conditional instability and triggered convection in a moist atmosphere with the minimum recipes.

Because of the simplicity of our model, we have to make assumptions about the MJO vertical structure and the Kelvin wave speed. Although these assumptions are consistent with observations, a complete theory will have to explain why the MJO has a first baroclinic structure, and why the Kelvin wave speed is about $16 \mathrm{~m} \mathrm{~s}^{-1}$. The next step is to use a 3D model to test our hypothesis. We do not need to assume the MJO vertical structure and the Kelvin wave speed in the 3D model. To keep the key features of our current model, we will implement triggered convection in the 3D model. In this model, CAPE will be accumulated until convective inhibition falls below a certain value close to zero, thus making it more difficult for convection to occur. This allows a large amount of CAPE to accumulate before convection occurs. 
The IG wave signal in our power spectrum is stronger than that reported in the observations (e.g., Fig. 1 of Kiladis et al.2009). This could be due to the simplicity of our convection scheme. It is also likely due to the rectification by clouds. Clouds can spread and cover the IG waves, such that they do not show up clearly in the distributions of cloudiness or brightness temperature. Therefore, their variability is reduced in the OLR power spectrum. Instead, one would like to use dynamical variables like the zonal wind field to test our hypotheses. The correlation between the MJO and the IG waves has already been examined using observational datasets (e.g., Yang and Ingersoll 2011; Yasunaga and Mapes 2012). Statistically significant correlations have been identified, but the IG waves can only explain very limited variance of the MJO. However, previous studies do not help to test our hypothesis. First, most of the previous studies used coarse temporal- and spatial-resolution datasets, so they cannot resolve high-frequency IG waves. A second problem is that these studies examined the correlations between the MJO and the WIG waves, and the MJO and the EIG waves separately. The interference pattern of the WIG and EIG waves are not even included. To test our hypothesis, one should first combine the WIG and EIG signals in a high-resolution dataset and identify their interference patterns. Then one should examine the correlations between the MJO and this identified interference pattern.

Acknowledgments. We thank two anonymous reviewers for their helpful comments. Da Yang was supported by the Earle C. Anthony Professor of Planetary Science Research Pool and the Division of Geological and Planetary Sciences Davidow Fund of the California Institute of Technology. He is currently supported by the Astronomy and Astrophysics Research Program of the National Science Foundation. We thank these organizations for their support.

\section{REFERENCES}

Andersen, J. A., and Z. Kuang, 2012: Moist static energy budget of MJO-like disturbances in the atmosphere of a zonally symmetric aquaplanet. J. Climate, 25, 2782-2804.

Arakawa, A., and W. Schubert, 1974: Interaction of a cumulus cloud ensemble with the large-scale environment, Part I. J. Atmos. Sci., 31, 674-701.

Benedict, J. J., and D. A. Randall, 2009: Structure of the MaddenJulian oscillation in the superparameterized CAM. J. Atmos. Sci., 66, 3277-3296.

Biello, J. A., and A. J. Majda, 2005: A new multiscale model for the Madden-Julian oscillation. J. Atmos. Sci., 62, 1694-1721.

Bretherton, C. S., P. N. Blossey, and M. Khairoutdinov, 2005: An energy-balance analysis of deep convective self-aggregation above uniform SST. J. Atmos. Sci., 62, 4273-4292.
Emanuel, K., 2007: Quasi-equilibrium dynamics of the tropical atmosphere. The Global Circulation of the Atmosphere, T. Schneider and A. H. Sobel, Eds., Princeton University Press, 186-218.

_ - J. Neelin, and C. Bretherton, 1994: On large-scale circulations in convecting atmospheres. Quart. J. Roy. Meteor. Soc., 120, 1111-1143.

Fuchs, Ž., and D. J. Raymond, 2002: Large-scale modes of a nonrotating atmosphere with water vapor and cloud-radiation feedbacks. J. Atmos. Sci., 59, 1669-1679.

_ and _ 2005: Large-scale modes in a rotating atmosphere with radiative-convective instability and WISHE. J. Atmos. Sci., 62, 4084-4094.

- and - 2007: A simple, vertically resolved model of tropical disturbances with a humidity closure. Tellus, 59A, 344354, doi:10.1111/j.1600-0870.2007.00230.x.

Hendon, H. H., and B. Liebmann, 1994: Organization of convection within the Madden-Julian oscillation. J. Geophys. Res., 99D, 8073-8083.

_- and M. L. Salby, 1994: The life cycle of the Madden-Julian oscillation. J. Atmos. Sci., 51, 2225-2237.

Holloway, C. E., and J. D. Neelin, 2007: The convective cold top and quasi equilibrium. J. Atmos. Sci., 64, 1467-1487.

, S. J. Woolnough, and G. M. S. Lister, 2012: Precipitation distributions for explicit versus parametrized convection in a large-domain high-resolution tropical case study. Quart. J. Roy. Meteor. Soc., 138, 1692-1708, doi:10.1002/qj.1903.

Khairoutdinov, M. F., and D. A. Randall, 2001: A cloud resolving model as a cloud parameterization in the NCAR community climate system model: Preliminary results. Geophys. Res. Lett., 28, 3617-3620.

Khouider, B., Y. Han, A. J. Majda, and S. N. Stechmann, 2012: Multiscale waves in an MJO background and convective momentum transport feedback. J. Atmos. Sci., 69, 915-933.

Kiladis, G. N., K. H. Straub, and P. T. Haertel, 2005: Zonal and vertical structure of the Madden-Julian oscillation. J. Atmos. Sci., 62, 2790-2809.

- M. C. Wheeler, P. T. Haertel, K. H. Straub, and P. E. Roundy, 2009: Convectively coupled equatorial waves. Rev. Geophys., 47, RG2003, doi:10.1029/2008RG000266.

Lin, J.-L., and Coauthors, 2006: Tropical intraseasonal variability in 14 IPCC AR4 climate models. Part I: Convective signals. J. Climate, 19, 2665-2690.

Madden, R. A., and P. R. Julian, 1972: Description of global-scale circulation cells in the tropics with a 40-50 day period. J. Atmos. Sci., 29, 1109-1123.

— oscillation-A review. Mon. Wea. Rev., 122, 814-837.

$\longrightarrow$, and - 2005: Historical perspective. Intraseasonal Variability in the Atmosphere-Ocean Climate System, W. K. M. Lau and D. E. Waliser, Eds., Springer, 1-18.

Majda, A. J., and S. N. Stechmann, 2009: The skeleton of tropical intraseasonal oscillations. Proc. Natl. Acad. Sci. USA, 106, $8417-8422$

— in the MJO skeleton. J. Atmos. Sci., 68, 3053-3071.

Maloney, E. D., 2009: The moist static energy budget of a composite tropical intraseasonal oscillation in a climate model. J. Climate, 22, 711-729.

Matsuno, T., 1966: Quasi-geostrophic motions in the equatorial area. J. Meteor. Soc. Japan, 44, 25-43.

Moncrieff, M. W., 2004: Analytic representation of the large-scale organization of tropical convection. J. Atmos. Sci., 61, 15211538. 
Nakazawa, T., 1988: Tropical super clusters within intraseasonal variations over the western Pacific. J. Meteor. Soc. Japan, 66, 823-839.

Neelin, J. D., and J.-Y. Yu, 1994: Modes of tropical variability under convective adjustment and the Madden-Julian oscillation. Part I: Analytical theory. J. Atmos. Sci., 51, 1876-1894.

Raymond, D. J., and Ž. Fuchs, 2009: Moisture modes and the Madden-Julian oscillation. J. Climate, 22, 3031-3046.

Sobel, A. H., J. Nilsson, and L. M. Polvani, 2001: The weak temperature gradient approximation and balanced tropical moisture waves. J. Atmos. Sci., 58, 3650-3665.

Solodoch, A., W. R. Boos, Z. Kuang, and E. Tziperman, 2011: Excitation of intraseasonal variability in the equatorial atmosphere by Yanai wave groups via WISHE-induced convection. J. Atmos. Sci., 68, 210-225.

Tung, W.-W., and M. Yanai, 2002: Convective momentum transport observed during the TOGA COARE IOP. Part I: General features. J. Atmos. Sci., 59, 1857-1871.

Wang, B., 2005: Theories. Intraseasonal Variability in the Atmosphere-Ocean Climate System, W. K. M. Lau and D. E. Waliser, Eds., Springer, 307-360.
Wheeler, M., and G. N. Kiladis, 1999: Convectively coupled equatorial waves: Analysis of clouds and temperature in the wavenumber-frequency domain. J. Atmos. Sci., 56, 374399.

_ , and H. H. Hendon, 2004: An all-season real-time multivariate MJO index: Development of an index for monitoring and prediction. Mon. Wea. Rev., 132, 1917-1932.

Xu, K.-M., and K. A. Emanuel, 1989: Is the tropical atmosphere conditionally unstable? Mon. Wea. Rev., 117, 14711479.

Yang, D., and A. P. Ingersoll, 2011: Testing the hypothesis that the MJO is a mixed Rossby-gravity wave packet. J. Atmos. Sci., 68, 226-239.

Yasunaga, K., and B. Mapes, 2012: Differences between more divergent and more rotational types of convectively coupled equatorial waves. Part II: Composite analysis based on space-time filtering. J. Atmos. Sci., 69, $17-34$.

Zhang, C., 2005: Madden-Julian oscillation. Rev. Geophys., 43, RG2003, doi:10.1029/2004RG000158. 